\title{
Análise calorimétrica de pastas de cimento Portland contendo polímero superabsorvente (SAP) e nanopartículas de sílica (NS)
}

\author{
A calorimetry analysis of Portland cement \\ pastes containing superabsorbent polymer \\ (SAP) and nanosilica (NS)
}

Lívia Borba Agostinho ${ }^{1}$, Juliana Gonçalves Borges ${ }^{1}$, Eugênia Fonseca da Silva ${ }^{1}$, Daiane Vitória Machado Ramos Cupertino ${ }^{2}$

\footnotetext{
${ }^{1}$ Universidade de Brasília, Programa de Pós-graduação em Estruturas e Construção Civil, Prédio SG-12, ${ }^{\circ}$ andar, Campus Darcy Ribeiro, Universidade de Brasília, CEP: 70910-900, Brasília, DF, Brasil.

${ }^{2}$ Furnas Centrais Elétricas, Centro Tecnológico de Engenharia Civil, BR-153, s/n, Zona Rural, CEP: 74923-650, Aparecida de Goiânia, GO, Brasil

e-mail: liviaborbagostinho@gmail.com, juliana.gborges@hotmail.com, eugenia@unb.br,dvmramos@gmail.com
}

\section{RESUMO}

A aplicação da técnica de calorimetria por condução isotérmica possibilita a avaliação da velocidade das reações de hidratação de forma simplificada e eficiente, ao longo do tempo, pelas curvas de evolução de calor. Essa técnica pode ser a ferramenta adequada para a avaliação do processo de dessorção do polímero superabsorvente (SAP), parâmetro fundamental que determina a eficiência do polímero como agente mitigador da retração autógena. Com o uso da calorimetria foi possível avaliar o comportamento do SAP durante o estado fresco e a mudança que a nanosílica (NS) causou na cinética de hidratação dos compostos cimentícios. Nas pastas estudadas nesse trabalho, observou-se um leve retardo na ocorrência do pico principal de hidratação do $\mathrm{C}_{3} \mathrm{~S}$ nas pastas contendo SAP e uma aceleração das reações de hidratação das pastas contendo NS. Notou-se ainda que quanto maior a quantidade de SAP e de NS, maior foi a quantidade de calor liberado, o que pode ser correlacionado com maior formação de hidratos.

Palavras-chave: calorimetria, hidratação, polímero superabsorvente, nanosílica.

\begin{abstract}
The application of calorimetry's technique by isothermal conduction allows the evaluation of the velocity of the hydration reactions in a simple and efficient way, over time, by the heat evolution's curves. This technique can be the right tool to evaluate the superabsorbent polymer's (SAP) desorption, which is a fundamental parameter that determines the polymer's efficiency as mitigating agent of autogenous shrinkage. With the use of calorimetry, it was possible to evaluate SAP's behavior during the fresh state and the influence of nanosilica (NS) in hydration kinetics of cement compounds. Regarding the pastes analyzed in this paper, it was observed a small retard in the main peak of C-S-H formation in the pastes containing SAP and an acceleration of the hydration's reactions of the pastes containing NS. It was also noted that the heat released is proportional to the amount of NS and SAP, what can be related to the higher hydrate's formation.
\end{abstract}

Keywords: calorimetry, hydration, superabsorbent polymer, nanosilica.

\section{INTRODUÇÃO}

Nas últimas décadas, com os avanços alcançados no projeto de estruturas de concreto armado tem-se buscado estruturas mais esbeltas e mais leves, e o concreto de alta resistência (CAR) é uma alternativa adequada para este fim. O princípio básico para sua obtenção está fundamentado na diminuição da porosidade, por meio da modificação da estrutura dos poros, que só foi possível devido à diminuição da relação água/cimento (a/c), 
graças ao uso de aditivos superplastificantes; à utilização de adições minerais; e à otimização da granulometria dos agregados [1].

$\mathrm{O}$ uso de adições minerais no $\mathrm{CAR}$, além do bom resultado relacionado à durabilidade, também proporciona às estruturas um melhor desempenho. $\mathrm{O}$ excelente resultado na atividade pozolânica de alguns desses materiais fez com que fossem denominadas adições minerais altamente reativas. Atualmente, como principais pozolanas altamente reativas têm-se a sílica ativa, o metacaulim e a cinza da casca de arroz com queima controlada [2].

Nessa perspectiva tem-se mais recentemente o uso da nanosílica (NS), partículas amorfas de dióxido de silício $\left(\mathrm{SiO}_{2}\right)$, não porosas, em escala nanométrica, que participam de forma mais efetiva das reações pozolânicas, resultando em materiais com desempenho superior àqueles sem a utilização de nanopartículas [2, 3].

Para os CAR, cuja compacidade da matriz cimenticia é maior que a dos concretos usuais, a cura externa tem pouco efeito na redução da retração autógena. A cura interna, no entanto, pode compensar o fraco papel da cura externa na sua fissuração precoce [4]. O polímero superabsorvente (SAP) é considerado o mais eficiente agente regulador de água, uma vez que tem a capacidade de absorver uma quantidade significativa de líquido a partir do ambiente e de reter este líquido dentro da sua estrutura, sem se dissolver, até que haja uma queda na umidade relativa e o líquido seja então liberado [5]. No meio cimentício, a liberação da água pelo polímero mantém os capilares saturados e promove a formação de novos hidratos, dado que essa água de cura interna também participa das reações de hidratação [6, 7].

A calorimetria de condução isotérmica quantifica diretamente o calor liberado na hidratação do cimento por meio do monitoramento do fluxo de calor da pasta. Esta técnica vem sendo amplamente utilizada para monitorar a cinética de hidratação e quantificar os produtos de hidratação [8]. Devido ao fornecimento de medidas contínuas, é um método versátil e conveniente para estudar o estágio inicial da hidratação de sistemas cimentícios, onde a taxa de calor é relativamente alta $[9,10]$. Este método pode ser a ferramenta adequada para a avaliação do processo de dessorção do polímero superabsorvente (SAP) e da mudança que a nanosílica (NS) causa na cinética de hidratação dos compostos cimentícios. Apenas seis artigos foram encontrados na literatura técnica sobre a utilização da calorimetria isotérmica em materiais cimentícios modificados com SAP [6, 11- 15].

Na Figura 1 é apresentada uma curva típica de evolução de calor da hidratação de uma pasta de cimento Portland, onde a cinética de hidratação é dividida em estágios.

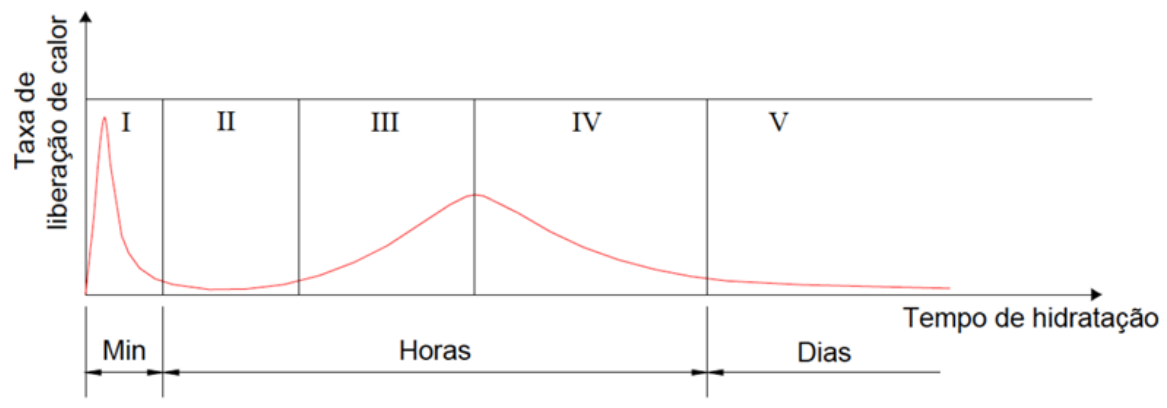

Figura 1: Representação gráfica da curva de evolução de calor gerada durante as reações de hidratação de uma pasta de cimento Portland.

O estágio I, de dissolução inicial, acontece nos primeiros minutos, nesta fase, apresenta-se o primeiro pico da evolução do calor de hidratação, cuja principal reação responsável é a dissolução do aluminato tricálcico $\left(\mathrm{C}_{3} \mathrm{~A}\right)$ e do sulfato de cálcio $\left(\mathrm{CaSO}_{4}\right)$, formando etringita $(\mathrm{AFt})$. O período II, de indução (ou dormência), tem algumas horas de duração e com taxa de hidratação baixa. O estágio III, de aceleração, é responsável pelo segundo pico exotérmico. Nesta fase, o silicato tricálcico $\left(\mathrm{C}_{3} \mathrm{~S}\right)$ e o silicato dicálcico $\left(\mathrm{C}_{2} \mathrm{~S}\right)$ reagem com a água e formam rapidamente o silicato de cálcio hidratado amorfo $(\mathrm{C}-\mathrm{S}-\mathrm{H})$ e o hidróxido de cálcio $\left(\mathrm{Ca}(\mathrm{OH})_{2}\right)$. Nesta ocorre a pega do cimento. O período IV, de desaceleração, inicia-se com a redução da taxa de calor. Finalmente, ocorre o estágio V, de reação lenta, associado com a transformação de AFt em Monossultafo (AFm) $[6,16]$.

Para realização do ensaio pode-se optar por fazer a mistura das pastas dentro do próprio calorímetro ou externo a ele. As duas opções se diferenciam na homogeneização das misturas e na aquisição dos dados iniciais de liberação de calor, uma vez que a mistura interna permite que se avalie toda a liberação de calor, desde 
os primeiros instantes da hidratação. Porém, a mistura interna é realizada a partir do misturador interno do equipamento, cuja velocidade máxima é de apenas $20 \mathrm{rpm}$, insuficiente para uma dispersão eficiente. A mistura externa, embora garanta uma homogeneização mais eficiente, perde a coleta de informações do estágio I (Figura 1), pois quando a mistura é introduzida no equipamento, já estão ocorrendo as reações de hidratação da mistura, o que impossibilita a aquisição de dados do primeiro pico de liberação de calor, com informações predominantemente sobre a reação dos aluminatos.

Como não se tem interesse específico nas reações dos aluminatos e a mistura externa confere maior homogeneidade das misturas contendo SAP e, das especialmente contendo NS, e por consequência, a confiabilidade dos resultados, optou-se por realizar mistura externa.

Este trabalho visa empregar a técnica de calorimetria por condução isotérmica para avaliar o efeito da adição de NS e SAP na cinética de hidratação dos compostos cimentícios.

\section{MATERIAIS E MÉTODOS}

\subsection{Materiais}

Para a realização deste estudo foi utilizado o cimento CPV-ARI fornecido pela CIPLAN, cujas caracterizações físicas, mecânicas e químicas estão apresentadas nas Tabela 1 e 2.

Tabela 1: Caracterização física e mecânica do cimento CPV ARI utilizado nesta pesquisa.

\begin{tabular}{|c|c|c|c|c|}
\hline \multicolumn{2}{|c|}{ PROPRIEDADES DETERMINADAS } & \multirow{2}{*}{$\frac{\text { CP V - ARI }}{0,4}$} & \multirow{2}{*}{$\begin{array}{c}\text { LIMITAÇÕES: } \\
\text { NBR 16697:2018 [17] } \\
\leq 6,0\end{array}$} & \multirow{2}{*}{$\begin{array}{l}\text { MÉTODO DE ENSAIO } \\
\text { NBR } 11579 / 2012 \text { [18] }\end{array}$} \\
\hline Finura & Retido na \# $200(\%)$ & & & \\
\hline \multicolumn{2}{|c|}{ Área específica $\left(\mathrm{cm}^{2} / \mathrm{g}\right)$ Blaine } & 5.440 & Não especificado & NBR 16372/2015 [19] \\
\hline \multirow{2}{*}{ Tempos de Pega } & Início (h:min) & 02:30 & $\geq 1: 00$ & NBR 16607/2018 [20] \\
\hline & Fim (h:min) & 03:10 & $\leq 10: 00$ & NBR 16607/2018 [20] \\
\hline \multirow{3}{*}{$\begin{array}{l}\text { Resistência à } \\
\text { Compressão }\end{array}$} & 1 dia $(\mathrm{MPa})$ & 32,1 & $\geq 14,0$ & NBR 7215/2019 [21] \\
\hline & 3 dias (MPa) & 37,2 & $\geq 24,0$ & NBR 7215/2019 [21] \\
\hline & 7 dias $(\mathrm{MPa})$ & 41,8 & $\geq 34,0$ & NBR 7215/2019 [21] \\
\hline
\end{tabular}

Tabela 2: Caracterização química do cimento CPV ARI utilizado nesta pesquisa (expressa em porcentagem de massa).

\begin{tabular}{|c|c|c|c|c|}
\hline \multicolumn{2}{|c|}{ PROPRIEDADES DETERMINADAS } & CP V - ARI & $\begin{array}{c}\text { LIMITAÇÕES: } \\
\text { NBR 16697:2018 [17] }\end{array}$ & MÉTODO DE ENSAIO \\
\hline \multicolumn{2}{|c|}{ Perda ao fogo $(\%)$} & 1,82 & $\leq 6,5$ & NBR NM 18/2012 [22] \\
\hline \multicolumn{2}{|c|}{ Resíduo insolúvel (\%) } & 0,79 & $\leq 3,5$ & NBR NM 15/2012 [23] \\
\hline \multicolumn{2}{|c|}{ Trióxido de enxofre $\left(\mathrm{SO}_{3}\right)(\%)$} & 3,28 & $\leq 4,5$ & NBR NM 16/2012 [24] \\
\hline \multicolumn{2}{|c|}{ Óxido de magnésio $(\mathrm{MgO})(\%)$} & 4,36 & $\leq 6,5$ & NBR NM 11-2/2012 [25] \\
\hline \multicolumn{2}{|c|}{ Dióxido de silício $\left(\mathrm{SiO}_{2}\right)(\%)$} & 24,41 & Não especificado & NBR NM 11-2/2012 [25] \\
\hline \multicolumn{2}{|c|}{ Óxido de ferro $\left(\mathrm{Fe}_{2} \mathrm{O}_{3}\right)(\%)$} & 3,02 & Não especificado & NBR NM 11-2/2012 [25] \\
\hline \multicolumn{2}{|c|}{ Óxido de alumínio $\left(\mathrm{Al}_{2} \mathrm{O}_{3}\right)(\%)$} & 7,09 & Não especificado & NBR NM 11-2/2012 [25] \\
\hline \multicolumn{2}{|c|}{ Óxido de cálcio $(\mathrm{CaO})(\%)$} & 53,74 & Não especificado & NBR NM 11-2/2012 [25] \\
\hline \multicolumn{2}{|c|}{ Óxido de cálcio livre $(\mathrm{CaO})(\%)$} & 2,16 & Não especificado & NBR NM 13/2013 [26] \\
\hline \multirow{3}{*}{$\begin{array}{l}\text { Álcalis } \\
\text { Totais (\%) }\end{array}$} & Óxido de sódio $\left(\mathrm{Na}_{2} \mathrm{O}\right)$ & 0,29 & Não especificado & NBR NM 17/2012 [27] \\
\hline & Óxido de potássio $\left(\mathrm{K}_{2} \mathrm{O}\right)$ & 0,77 & Não especificado & NBR NM 17/2012 [27] \\
\hline & Equiv. alcalino & 0,80 & Não especificado & NBR NM 17/2012 [27] \\
\hline \multirow{3}{*}{$\begin{array}{l}\text { Álcalis } \\
\text { Solúveis } \\
\text { em Água (\%) }\end{array}$} & Óxido de sódio $\left(\mathrm{Na}_{2} \mathrm{O}\right)$ & 0,18 & Não especificado & NBR NM 17/2012 [27] \\
\hline & Óxido de potássio $\left(\mathrm{K}_{2} \mathrm{O}\right)$ & 0,64 & Não especificado & NBR NM 17/2012 [27] \\
\hline & Equiv. alcalino & 0,60 & Não especificado & NBR NM 17/2012 [27] \\
\hline \multicolumn{2}{|c|}{ Sulfato de cálcio $\left(\mathrm{CaSO}_{4}\right)(\%)$} & 5,57 & Não especificado & NBR NM 16/2012 [24] \\
\hline
\end{tabular}


As caracterizações químicas e físicas do cimento foram realizadas segundo os métodos de ensaio citados na Tabela 2, cujos valores se apresentaram dentro dos limites previstos pela norma de requisitos ABNT NBR 16697:2018 [17]. É importante salientar o baixo teor de resíduo insolúvel e não se constatou evidência de material pozolânico. Os teores de óxido de cálcio livre e óxido de magnésio estão em níveis aceitáveis, o que provavelmente não irá causar reação expansiva do cimento, devido à hidratação tardia destes compostos $(\mathrm{CaO}$ e $\mathrm{MgO})$.

A nanosílica $\left(\mathrm{SiO}_{2}\right)$ utilizada nesta pesquisa é a Levasil CB30, uma solução aquosa de sílica coloidal, com teor de sólidos de 30\% fornecida pela Akzonobel. As características da nanosílica foram fornecidas pelo fabricante e estão apresentadas na Tabela 3. O amorfismo das partículas de nanosílica foi confirmado pela realização de difração de raios-x, conforme mostrado na Figura 2, pela apresentação do halo amorfo e ausência de picos de compostos cristalinos que foram determinados em um difratometro de raio-X operando com um filamento de tungstênio como cátodo e um tubo de raio-X de cobre, sob $35 \mathrm{kV}$ e $15 \mathrm{~mA}$, no intervalo de $2 \theta$ igual a $2^{\circ}$ até $60^{\circ}$ com velocidade de varredura de $5^{\circ} / \mathrm{min}$ e passos de $0,5^{\circ}$.

Tabela 3: Características da nanosílica utilizada neste trabalho de acordo com o fabricante.

\begin{tabular}{c|c}
\hline Natureza química & Sílica amorfa \\
\hline Apresentação - estado físico & Solução aquosa coloidal - líquido \\
\hline Cor & Claro (ligeiramente turvo) \\
\hline Odor & Suave \\
\hline Teor de sílica & $30 \%$ \\
\hline Tamanho aproximado das partículas & $\sim 5 \mathrm{~nm}$ \\
\hline Área superficial & $300 \mathrm{~m}^{2} / \mathrm{g}$ \\
\hline pH & $10,5(9-11)$ \\
\hline Viscosidade & $<50 \mathrm{MPa} \cdot \mathrm{s}$ \\
\hline Densidade & $1,2 \mathrm{~g} / \mathrm{cm}^{3}$ \\
\hline Teor $\mathrm{NaO}_{2}$ & $0,55 \%$ \\
\hline
\end{tabular}

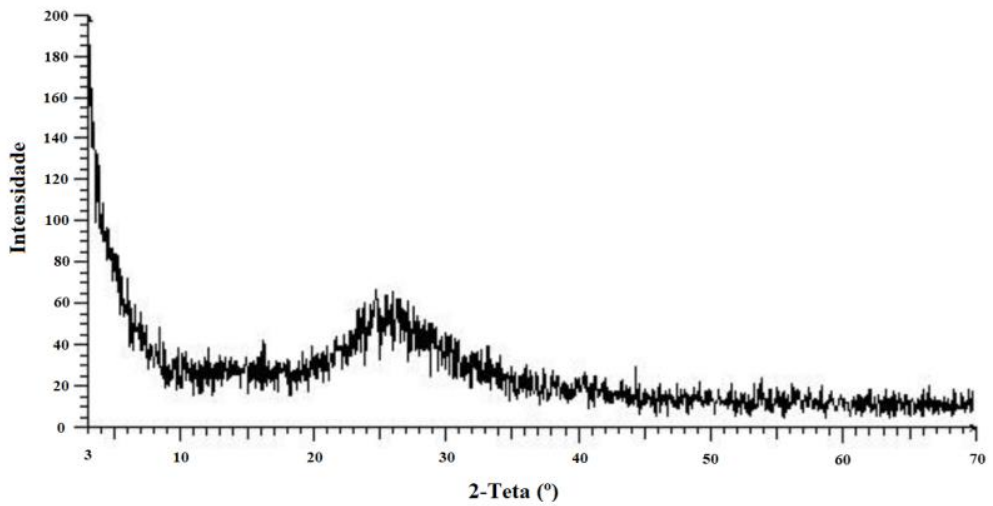

Figura 2: Perfil difratométrico da nanosílica empregada no estudo.

$\mathrm{O}$ aditivo superplastificante utilizado foi à base de eterpolicarboxilato, sendo um líquido viscoso branco turvo, com massa específica entre $1,067 \mathrm{~g} / \mathrm{cm}^{3}$ e $1,107 \mathrm{~g} / \mathrm{cm}^{3}$ e teor de sólidos de $31,5 \%$. Os dados foram fornecidos pela fabricante BASF.

O SAP utilizado é um ácido acrílico/acrilamida com ligações cruzadas covalentes, produzido pela técnica de polimerização por suspensão inversa e foi desenvolvido para uso especial em ambiente alcalino elevado, como os materiais cimentícios [28] desenvolvido e doado pelo professor Ole Mejlhede Jensen, da Denmark Technical University (DTU). Na Tabela 4 é apresentado um resumo da caracterização do polímero superabsorvente utilizado nesta pesquisa, realizada por NETO [29] e MANZANO [6]. 
Tabela 4: Características do Polímero Superabsorvente [6, 29].

\begin{tabular}{c|c}
\hline Composição química & C, O, Na, S \\
\hline Forma das partículas & Esférica \\
\hline Processo de produção & Suspensão inversa \\
\hline Massa específica & $1,456 \mathrm{~g} / \mathrm{cm}^{3}$ \\
\hline Diâmetro seco & $66,3 \mu \mathrm{m}$ \\
\hline Diâmetro inchado & $189,6 \mu \mathrm{m}$ \\
\hline Absorção em meio aquoso & $95,8 \mathrm{~g} / \mathrm{g}$ \\
\hline Absorção em meio cimentício & $15,0 \mathrm{~g} / \mathrm{g}$ \\
\hline
\end{tabular}

Conforme mostrado na Tabela 4, a absorção do polímero em meio cimentício é de $15 \mathrm{~g} / \mathrm{g}$, ou seja, cada grama de polímero seco absorve 15 gramas de água, em meio cimentício, determinado pela técnica de espalhamento de argamassa [6, 29]. Este dado é de especial importância na dosagem das pastas e foi utilizado na determinação da quantidade de água de cura interna (absorvida pelo SAP) adicionada à mistura $\left(\mathrm{a} / \mathrm{c}_{\mathrm{i}}\right)$.

\subsection{Métodos}

Seguindo a recomendação do comitê da RILEM TC 225 SAP [30], utilizou-se a mesma relação a/c básica $\left(\mathrm{a} / \mathrm{c}_{\mathrm{b}}\right)$ para todas as misturas, de modo que nas misturas contendo SAP, a relação $\mathrm{a} / \mathrm{c}$ total $\left(\mathrm{a} / \mathrm{c}_{\mathrm{t}}\right)$ foi maior em virtude da adição da água de cura interna $\left(\mathrm{a} / \mathrm{c}_{\mathrm{t}}=\mathrm{a} / \mathrm{c}_{\mathrm{b}}+\mathrm{a} / \mathrm{c}_{\mathrm{i}}\right)$.

A matriz experimental adotada nesta pesquisa está apresentada na Tabela 5. Nove pastas foram estudadas, formando uma matriz quadrada $3 \times 3$, variando-se os teores de SAP $(0,0,15 \%$ e $0,30 \%)$ e os teores de nanosílica $(0,1 \%$ e $2 \%)$. A relação água/cimento básica $\left(\mathrm{a} / \mathrm{c}_{\mathrm{b}}\right)$ foi fixada em 0,4 , assim como o teor de aditivo superplastificante (SP), fixado em 1,2\% em relação a massa de cimento. Foi produzido um volume de 150 gramas de cada pasta. Na Tabela 5 estão apresentados os valores de $\mathrm{a} / \mathrm{c}_{\mathrm{t}}$, de todas as pastas produzidas nesse estudo.

Tabela 5: Matriz de dosagem experimental.

\begin{tabular}{l|c|c|c}
\hline NOMENCLATURA DO TRAÇO & TEOR DE NS (\%) & TEOR DE SAP (\%) & A/C TOTAL \\
\hline REF & 0,00 & 0,00 & 0,40 \\
\hline NS1 & 1,00 & 0,00 & 0,40 \\
\hline NS2 & 2,00 & 0,00 & 0,40 \\
\hline SAP15 & 0,00 & 0,15 & 0,43 \\
\hline SAP15 NS1 & 1,00 & 0,15 & 0,43 \\
\hline SAP15 NS2 & 2,00 & 0,15 & 0,43 \\
\hline SAP30 & 0,00 & 0,30 & 0,45 \\
\hline SAP30 NS1 & 1,00 & 0,30 & 0,45 \\
\hline SAP30 NS2 & 2,00 & 0,30 & 0,45 \\
\hline
\end{tabular}

\subsubsection{Mistura}

Após a pesagem dos materiais, o SAP foi misturado ao cimento manualmente, com o auxílio de uma espátula, para evitar o aparecimento do efeito gel bloking [31]. Em seguida, os materiais secos foram transferidos para um béquer para posterior adição dos líquidos, previamente misturados (água, aditivo superplastificante e nanosílica coloidal) em um misturador mecânico com velocidade de $2500 \mathrm{rpm}$ para proporcionar uma melhor dispersão da nanosílica e ativação do aditivo superplastificante. A sequência do procedimento de mistura está esquematizada na Tabela 6. 
Tabela 6: Sequência do procedimento de mistura das pastas

\begin{tabular}{l|c|c}
\hline PROCEDIMENTO & TEMPO & TIPO DE MISTURA /VELOCIDADE \\
\hline Mistura materiais líquidos (água, NS e SP) & $2 \mathrm{~min}$ & Mecânico $-2500 \mathrm{rpm}$ \\
\hline Mistura líquidos e materiais secos & $2 \mathrm{~min}$ & Mecânico $-2500 \mathrm{rpm}$ \\
\hline Raspagem paredes béquer & $1 \mathrm{~min}$ & Manual \\
\hline Mistura final & $5 \mathrm{~min}$ & Mecânico $-2500 \mathrm{rpm}$ \\
\hline
\end{tabular}

\subsubsection{Ensaio de calorimetria por condução isotérmica}

Os ensaios de calorimetria por condução isotérmica foram realizados em um calorímetro de condução isotérmica Thermometric TAM AIR, composto de oito canais com controle de temperatura, fabricado pela $T A$ Instruments com as características apresentadas na Tabela 7 e com aquisição de dados feita pelo software PicoLog. O ensaio foi realizado a temperatura de $25^{\circ} \mathrm{C}$.

Tabela 7: Especificações do equipamento TAM AIR utilizado nos ensaios de calorimetria de condução isotérmica.

\begin{tabular}{c|c}
\hline Amplitude de temperatura & $\mathbf{5}^{\circ} \mathbf{C}$ a $\mathbf{9 0}{ }^{\circ} \mathbf{C}$ \\
\hline Tipo do termostato & Air \\
\hline Estabilidade do termostato & $\pm 0,02^{\circ} \mathbf{C}$ \\
\hline Capacidade máxima de amostra & $20 \mathrm{ml}$ \\
\hline Limite de detecção & $4 \mu \mathrm{W}$ \\
\hline Precisão de detecção & $\pm 20 \mu \mathrm{W}$ \\
\hline
\end{tabular}

O objetivo deste ensaio é avaliar a liberação de calor proveniente das reações químicas do contato do cimento com a água e demais adições, possibilitando analisar a influência do SAP e da nanosílica nas reações de hidratação das pastas de cimento estudadas. Optou-se pela realização da metodologia de mistura externa e posterior introdução das pastas nas ampolas e no calorímetro. A sequência de preparo das amostras externas e execução do ensaio está descrita a seguir:

1. Mistura das pastas externamente;

2. Introdução da pasta pronta na ampola e pesagem de sua quantidade;

3. Introdução das ampolas com as pastas e das ampolas de referência vazias nos canais;

4. Marcação do tempo decorrido do contato líquido-sólido até a introdução da ampola no calorímetro;

5. Cálculo da quantidade de cada componente a partir da massa da amostra inserida na ampola para cadastro dos componentes no software;

Na Figura 3 são apresentadas algumas das etapas citadas anteriormente.

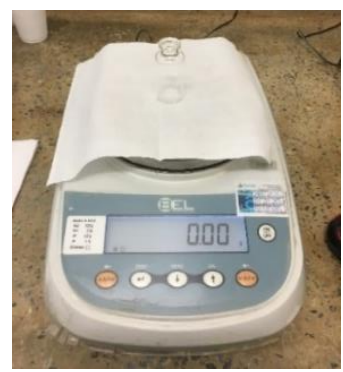

(a)

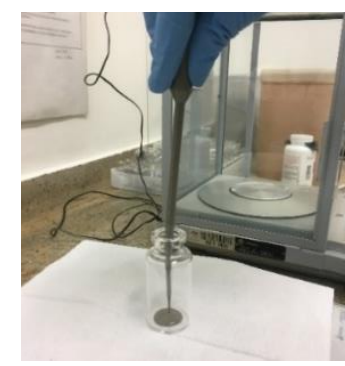

(b)

Figura 3: Processo de preparo das amostras e execução do ensaio externo: a) pesagem da ampola; b) introdução da pasta na ampola. 


\section{RESULTADOS E DISCUSSÃO}

Na Figura 4 são apresentadas as curvas de fluxo de calor liberado em função do tempo e na Figura 5 são apresentadas as curvas de liberação de calor acumulado para as nove pastas ensaiadas durante as 72 horas de ensaio.

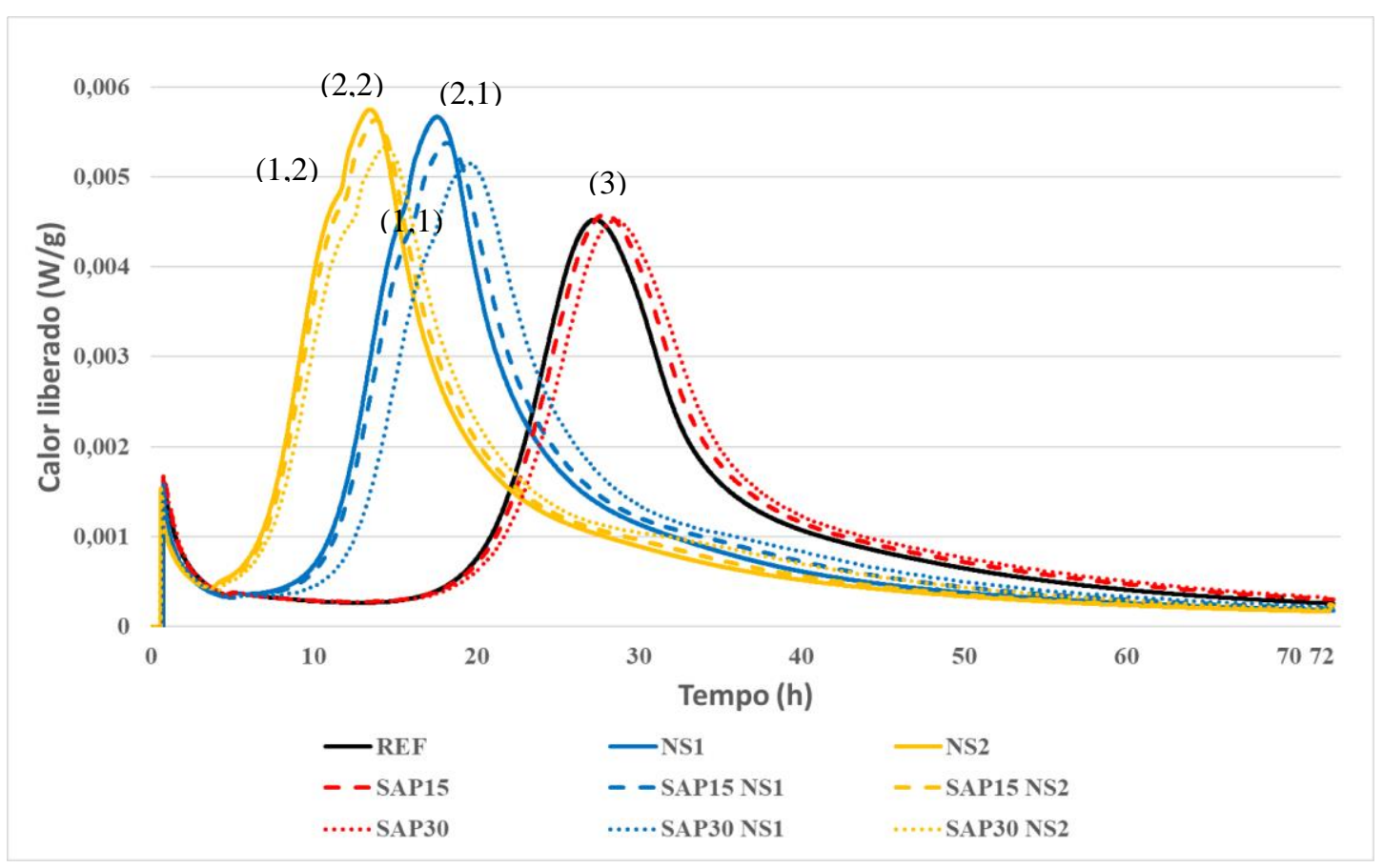

Figura 4: Curvas de fluxo de calor liberado em função do tempo (as numerações se referem aos picos de hidratação e serão discutidos nos subtópicos 3.1, 3.2 e 3.3).

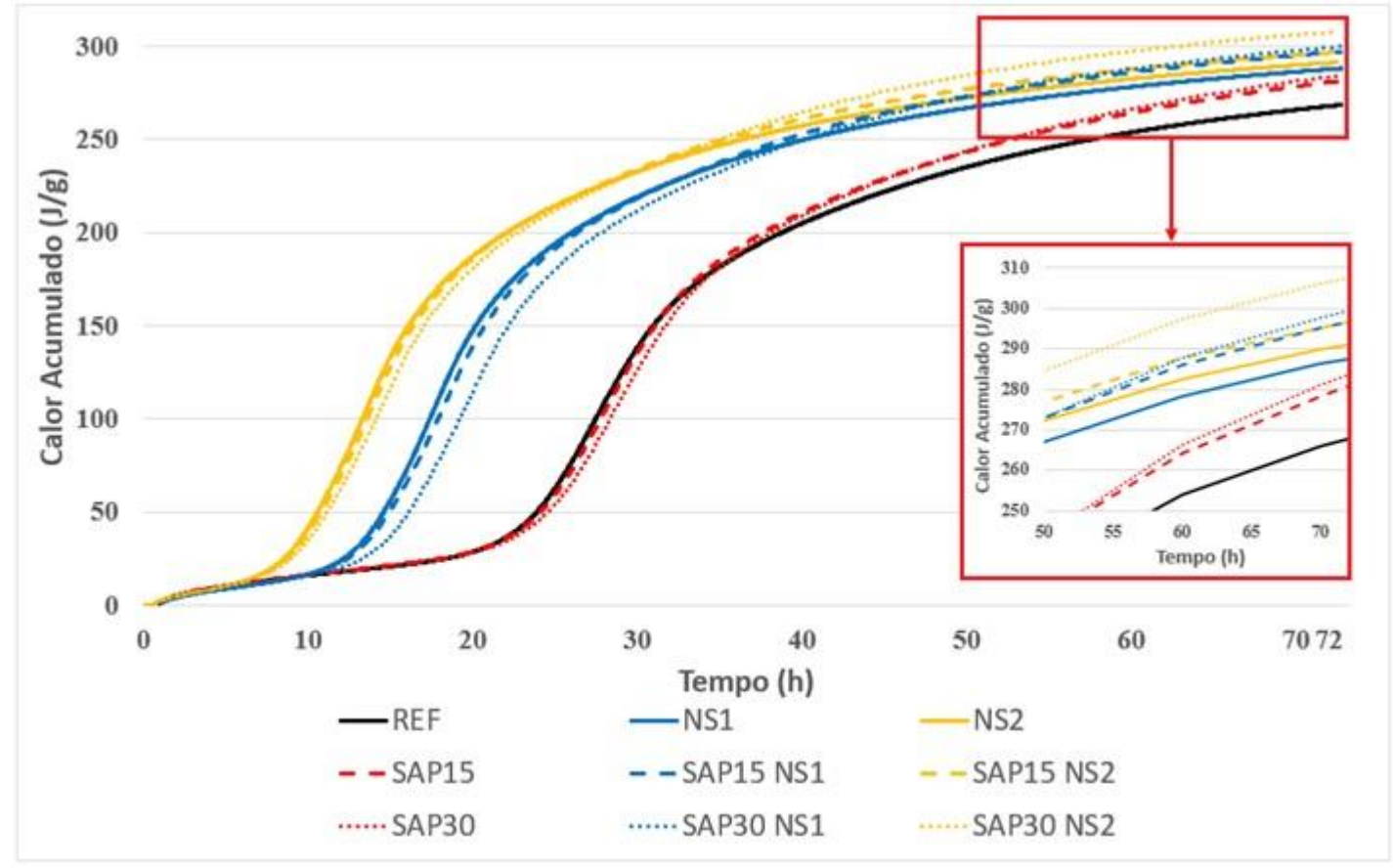

Figura 5: urvas de calor acumulado em função do tempo.

Além da apresentação gráfica das curvas de cinética de hidratação, foram calculados parâmetros calorimétricos como taxa de aceleração, quantidade de calor no pico de hidratação do $\mathrm{C}_{3} \mathrm{~S}$ e tempo do pico de hi- 
dratação do $\mathrm{C}_{3} \mathrm{~S}$ pelo método gráfico das tangentes como apresentado na Figura 6. A taxa de aceleração foi calculada a partir de uma regressão linear do segmento específico da curva de fluxo de calor em função do tempo em horas (valor do primeiro termo da equação da reta de ângulo $\alpha$ ). Os resultados calorimétricos obtidos pela análise gráfica pelo método das tangentes estão apresentados na Tabela 8.

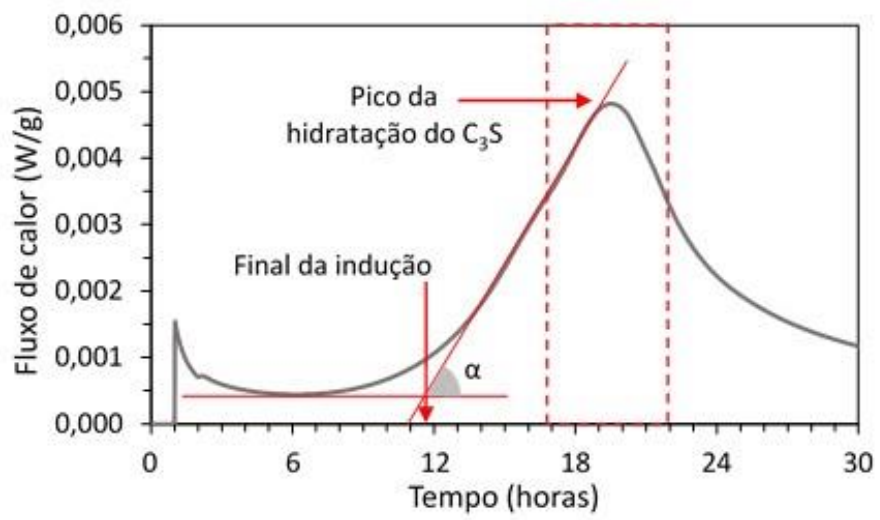

Figura 6: Ilustração do método gráfico das tangentes.

Tabela 8: Resumo dos parâmetros calculados do ensaio de calorimetria por condução isotérmica das pastas.

\begin{tabular}{l|c|c|c|c}
\hline PASTAS & $\begin{array}{c}\text { CALOR ACUMU- } \\
\text { LADO TOTAL (J/g) }\end{array}$ & $\begin{array}{c}\text { PICO DE HIDRA- } \\
\text { TAÇÃO } \mathbf{C}_{3} \mathbf{S}(\mathbf{m W} / \mathbf{g})\end{array}$ & $\begin{array}{c}\text { TEMPO DO PICO } \\
\text { DE HIDRATAÇÃO } \\
\mathbf{C}_{3} \mathbf{S} \mathbf{( h )}\end{array}$ & $\begin{array}{c}\text { TAXA DE ACELE- } \\
\text { RAÇÃO (\%o) }\end{array}$ \\
\hline REF & 268,5 & 4,5 & 27,3 & 0,78 \\
\hline NS1 & 288,1 & 5,7 & 17,6 & 0,97 \\
\hline NS2 & 291,3 & 5,7 & 13,5 & 1,08 \\
\hline SAP15 & 281,5 & 4,6 & 27,9 & 0,70 \\
\hline SAP15 NS1 & 297,1 & 5,4 & 18,2 & 0,93 \\
\hline SAP15 NS2 & 296,8 & 5,6 & 13,8 & 0,010 \\
\hline SAP30 & 284,4 & 4,5 & 28,6 & 0,88 \\
\hline SAP30 NS1 & 299,9 & 5,1 & 19,6 & 0,92 \\
\hline SAP30 NS2 & 352,7 & 5,3 & 14,5 & \\
\hline
\end{tabular}

Para melhor entendimento dos efeitos das adições do SAP e da NS na cinética de hidratação das pastas até os 3 dias de idade, a análise dos resultados apresentados anteriormente foi dividida em três tópicos, avaliando o comportamento calorimétrico das pastas contendo apenas NS, apenas SAP e pastas híbridas com ambas as adições.

\subsection{Comportamento calorimétrico das pastas contendo NS}

Observando a Figura 4, nota-se que o teor de NS tem grande influência no comportamento das curvas, uma vez que antecipou consideravelmente as reações de hidratação. O período de aceleração é o período das rápidas reações principais de formação de C-S-H e hidróxido de cálcio $\left(\mathrm{Ca}(\mathrm{OH})_{2}\right)$ e seu início pode ser associado ao tempo de início de pega [32].

Fica evidente que a presença de NS antecipou as reações de hidratação, como já era esperado, uma vez que as nano-partículas de sílica funcionam como pontos de nucleação e geram mais reações pozolânicas, formando mais C-S-H, e pela possível alteração na concentração íons de Ca, que tendem a acelerar as reações de hidratação, pois aumenta rapidamente a disposição dos íons cálcio e seu rápido consumo em reações pozolanicas [33]. A pasta NS1 teve seu pico de hidratação do $\mathrm{C}_{3} \mathrm{~S}$ (Figura 4 - pico 2.1) antecipado em quase 10 horas com relação à pasta de referência (Figura 4 - pico 3), enquanto a pasta NS2 obteve antecipação de cerca de 14 horas (Figura 4 - pico 2.2).

LOTHENBACH et al. [34] avaliaram curvas de calorimetria de pastas de cimento com alguns fíleres e 
concluíram que as pastas contendo fíler geraram mais calor durante a hidratação e que o aumento do pico referente à segunda hidratação de etringita (picos 2.1 e 2.2 da Figura 4) foi mais acentuado que os picos $1.1 \mathrm{e}$ 1.2 da mesma figura. Os autores afirmaram que o efeito de nucleação dessas partículas pode interferir de maneira acentuada na hidratação dos aluminatos e que um impacto mais significante pode ser observado na hidratação dos aluminatos do que dos silicatos.

Segundo ZUNINO e SCRIVENER [35, 36] adições minerais podem ter um impacto significativo no balanço de sulfato de compostos cimentícios devido ao seu efeito fíler e seu impacto está relacionado à área superficial adicional, e não à composição química do material. Os íons sulfato são adsorvidos no precipitado de C-S-H devido à hidratação contínua do $\mathrm{C}_{3} \mathrm{~S}$. $\mathrm{O}$ aumento na taxa de reação de $\mathrm{C}_{3} \mathrm{~S}$ devido ao efeito fíler da adição mineral ou da finura do $\mathrm{C}_{3} \mathrm{~S}$, acelera a taxa de precipitação de $\mathrm{C}-\mathrm{S}-\mathrm{H}$, aumentando a quantidade de sulfato adsorvido, e consequentemente acelera a ocorrência do pico de hidratação do C3S e da formação de etringita, confirmando o comportamento observado nas pastas contendo NS.

Para as pastas contendo $2 \%$ de NS também ficou claro, na Figura 4, o menor período de indução (3-4 horas) se comparado com as demais misturas, ou seja, curto tempo onde as reações de hidratação foram lentas. Com relação à intensidade do pico de liberação de calor $(2.1$ e 2.2$)$, para as pastas contendo 1 e $2 \%$ de $\mathrm{NS}$, esses foram praticamente iguais $(5,7 \mathrm{~mW} / \mathrm{g}$ em ambas as pastas com NS), porém têm desprendimento maior de calor (da ordem de $25 \%$ ), quando comparadas às pastas sem adição de NS $(4,5 \mathrm{~mW} / \mathrm{g}$ na pasta REF e SAP30 e 4,6 mW/g na pasta SAP15).

Em relação ao calor acumulado durante as 72 horas de ensaio (Figuras 5 e 6), o incremento deste calor está relacionado com a participação da adição nas reações de hidratação. Sabe-se que, quimicamente, a NS tem ação pozolânica, o que aumenta a formação de hidratos e o calor total liberado. De acordo com os valores apresentados na Figura 6 para as pastas REF (268,5 J/g), NS1 (288,1 J/g) e NS2 (291,3 J/g), percebe-se que a NS influenciou as reações de hidratação das pastas, uma vez que houve aumento do calor liberado a medida que se aumentou a quantidade de NS adicionada às misturas. Tal influência já era esperada, uma vez que a NS, além da reação pozolânica, contribui para as reações de hidratação, também fisicamente, com a formação de pontos de nucleação devido a sua elevada área superficial.

ANDRADE [2] e FRAGA [37], além da análise calorimétrica de pastas contendo nanopartículas de sílica, também fizeram ensaios de difratometria de raio X (DRX) e termogravimetria (TG) em pastas contendo NS em idades diversas. Ambos os autores comprovaram que as pastas contendo NS tem elevada capacidade em promover reações pozolânicas, pois diminuem consideravelmente a intensidade do pico de $\mathrm{Ca}(\mathrm{OH}) 2$ nos ensaios de $\mathrm{DRX}$ e diminuem do teor de $\mathrm{Ca}(\mathrm{OH}) 2$ em relação à pasta de referência para os ensaios de TG. Esses resultados corroboram com o comportamento observado nos ensaios de calorimetria executados nesta pesquisa.

\subsection{Comportamento calorimétrico das pastas contendo SAP}

Analisando agora o efeito do SAP isoladamente nas curvas de fluxo de calor (Figura 4), este gerou um leve retardo no tempo de ocorrência do pico calorimétrico (cerca de 10\%), quando comparadas com a pasta REF. Também a presença do SAP alterou ligeiramente a cinética de reação do cimento, reduzindo a taxa de aceleração da curva de liberação de calor.

As pastas contendo somente SAP não apresentaram picos de hidratação (1.1) e (1.2) como mostrado na Figura 4, apresentando somente o pico (3). Esse comportamento mostra mais uma vez a influência de adições minerais na cinética de hidratação de compostos cimentícios. A presença do SAP não interfere na formação de novos hidratos, pois somente fornece água extra para promover cura interna e hidratação.

Segundo KANG et al. [38], o uso da água de cura interna a partir do SAP pode aumentar o grau de hidratação dos compostos cimentícios. Segundo esses autores, a água de cura interna é liberada para o meio cimentício durante o período de aceleração, sendo consumida durante o período de desaceleração.

A respeito do calor acumulado (Figuras 5 e 6), percebe-se também um incremento do calor total com a adição do polímero. MANZANO [6] concluiu em seu trabalho que a água de cura interna participa das reações de hidratação, justificando os maiores valores de calor acumulado para as pastas com SAP. Nesse trabalho foi adicionada água extra de cura interna, aumentando assim a relação a/c total, com isso, espera-se que uma maior relação a/c promova mais reações de hidratação, justificando mais uma vez o aumento de calor total ao final de 3 dias de ensaio das pastas contendo SAP.

MANZANO [6], além dos ensaios calorimétricos também executou ensaios de DRX e TG das pastas contendo adição de 2 teores de SAP $(0,15 \%$ e $0,30 \%)$ e também considerando a/ $\mathrm{c}_{\mathrm{b}}$ iguais para todas as misturas (adicionando água extra nas pastas contendo SAP) e segundo o autor, foi constatado, pela análise termo- 
gravimétrica, que a adição de água de cura interna, incorporada pelo SAP, leva a um incremento do grau de hidratação da pasta, comprovando que a água de cura interna participa das reações de hidratação, corroborando com o aumento do calor total liberado nos ensaios calorimétricos desta pesquisa.

\subsection{Comportamento calorimétrico das pastas contendo NS e SAP}

É possível observar que a presença de NS aumentou a intensidade do pico da hidratação do $\mathrm{C}_{3} \mathrm{~S}$, enquanto que o SAP tendeu a diminuir a intensidade desse pico, de forma que nas misturas híbridas, a presença do SAP tende a minimizar esse incremento de calor causado pela presença da NS, mesmo assim, não sendo inferiores ao valor da pasta REF. Os maiores valores de liberação de calor na máxima hidratação de $\mathrm{C}_{3} \mathrm{~S}$ foram das pastas contendo somente NS ( 1 e $2 \%$ ), o que sugere a ocorrência do efeito fíler, gerando pontos de nucleação e consumo de $\mathrm{CH}$ para formação de mais C-S-H, resultados que corroboram com ANDRADE [2] e FRAGA [34].

O incremento do calor acumulado está relacionado com a participação da NS e SAP nas reações de hidratação. Como visto nos itens 3.1 e 3.2, ambas as adições promoveram incremento no calor acumulado isoladamente. E, conforme esperado, a combinação entre NS e SAP promoveu os maiores valores de calor total acumulado nas pastas híbridas, ou seja, tanto a presença de água extra de cura interna, quanto a presença de NS aumentam a formação de hidratos, seja pela disponibilidade maior de água para promover hidratação, seja pela presença de pontos de nucleação e agentes de reação pozolânica.

Ao final de 72 horas a pasta que apresentou maior valor de calor acumulado foi a SAP30NS2, contendo maiores teores de NS e SAP e água, consequentemente, maior relação a/c total, com isso maior possibilidade de formação de hidratos, tanto pela quantidade extra de água para hidratação, quanto pela maior quantidade de nanopartículas de sílica para as reações pozolânicas. Essa maior quantidade de água disponível para hidratação pode ter beneficiado as reações da NS, pois a pasta SAP30NS2 apresentou calor total acumulado maior do que as pastas SAP30 e NS2, onde SAP e NS atuam isoladamente. A pasta SAP30 apresentou calor total acumulado ao final do ensaio de $284,4 \mathrm{~J} / \mathrm{g}$ enquanto que a pasta NS2 apresentou valor de $291,3 \mathrm{~J} / \mathrm{g}$, isso mostra que adicionar NS é mais eficiente na formação de hidratos nos primeiros dias do que aumentar a relação a/c total (adicionar água extra de cura interna).

\section{CONCLUSÕES}

A presença de SAP alterou a cinética da hidratação do cimento, pois apresentaram menores picos de hidratação do $\mathrm{C}_{3} \mathrm{~S}$ e menores taxas de aceleração das reações, esse fato ocorreu ou pela ocorrência da cura interna (liberação de água após a redução da umidade relativa da matriz) ou pela presença de água extra que alterou a relação $\mathrm{a} / \mathrm{c}_{\mathrm{t}}$.

A presença da NS acelerou as reações de hidratação, seja pelo efeito fíler (gerando pontos de nucleação), ou pelo possível aumento da concentração iônica da pasta, e foi tal que quanto maior o teor da adição, menor a duração do período de dormência e mais cedo ocorreu a pega.

Para as pastas híbridas, observou-se que a presença de SAP e NS no teor mais elevado para ambas apresentou maior resultado de calor total acumulado, o que normalmente é correlacionado com a maior ocorrência de reações de hidratação (maior quantidade de água e aumento de pontos de nucleação). Tal fato mostra que a correlação dos dois materiais beneficia a formação de hidratos, pois o calor total liberado pelas pastas híbridas foi maior do que pelas duas pastas contendo SAP e NS isoladamente.

\section{AGRADECIMENTOS}

Os autores deste trabalho agradecem à Universidade de Brasília (UnB); ao laboratório e equipe de Furnas Centrais Elétricas S.A., onde os ensaios foram realizados; e ao Conselho Nacional de Desenvolvimento Científico e Tecnológico (CNPq), pelo apoio financeiro.

Agradecem também ao professor Ole Mejlhede Jense, da Denmark Technical University (DTU), pela doação do polímero superabsorvente; à empresa Akzonobel, pela doação da nanosílica; e à BASF, pela doação do aditivo superplastificante.

\section{BIBLIOGRAFIA}

[1] ISAIA, G.C., Concreto: Ciência e Tecnologia, 1ª. ed., São Paulo, IBRACON, 2011.

[2] ANDRADE, D.D.S., Microestrutura de pastas de cimento portland com nanossílica coloidal e adições minerais altamente reativas, Tese de Doutorado, PECC/UnB, Brasília, DF, Brasil, 2017. 
[3] SCRIVENER, K.L., KIRKPATRICK, R.J., "Innovation in use and research on cementitious material", Cement and Concrete Research, v. 38, pp. 128-136, Feb. 2008.

[4] WU, L., FARZADNIA, N.; SHI, C., et al., "Autogenous shrinkage of high performance concrete: A review”, Construction and Building Materials, v. 149, pp. 62-75, Sep. 2017.

[5] JENSEN, O. M., HANSEN, P. F., "Water-entrained cement-based materials I. Principles and theoretical background", Cement and Concrete Research, v. 31, pp. 647-654, Apr. 2001.

[6] MANZANO, M.A.R., Estudo Experimental de Materiais Cimentícios de Alta Resistência modificados com Polímeros Superabsorventes (PSAs) como Agentes de Cura Interna, Tese de doutorado, PECC/UnB, Brasília, DF, Brasil, 2016.

[7] HASHOLT, M.T., JENSEN, O.M., "Chloride migration in concrete with superabsorbent polymers", $\mathrm{Ce}$ ment and Concrete Composites, v. 55, p. 290-297, Jan. 2015.

[8] QUARCIONI, V.A., Influência da cal hidratada nas idades iniciais da hidratação do cimento Portland: estudo em pasta, Tese de doutorado, PPGEC/Poli/USP, São Paulo, SP, Brasil, 2008.

[9] ZHANG, G., ZHAO, J., WANG, P., et al., "Effect of HEMC on the early hydration of Portland cement highlighted by isothermal calorimetry", Journal of Thermal Analysis and Calorimetry, v. 119, pp. 1833-1843, Mar. 2015.

[10] ŠILER, P., KOLÁŘOVÁ, I., NOVOTNÝ, R., et al.,Journal of Thermal Analysis and Calorimetry, v. 133, pp. 27-40, Jul. 2018.

[11] ZHUTOVSKY, S., KOVLER, K. "Hydration kinetics of high-performance cementitious systems under different curing conditions". Materials and Structures. v. 46, pp.1599-1611. 2013.

[12] JUSTS, J., WYRZYKOWSKI, M., WINNEFELD, F., et al., "Influence of superabsorbent polymers on hydration of cement pastes with low water-to-binder ratio: A calorimetry study", Journal of Thermal Analysis and Calorimetry, v. 115, n. 1, pp. 425-432, 2014.

[13] JUSTS, J., WYRZYKOWSKI, M., BAJARE, D., et al., "Internal curing by superabsorbent polymers in ultra-high performance concrete". Cement and Concrete Research, v. 76, pp. 82-90, 2015.

[14] WANG, F. YANG, J., HU, S., et al., "Influence of superabsorbent polymers on the surrounding cement paste". Cement and Concrete Research, v. 81, pp. 112-121, 2016.

[15] AGOSTINHO, L.B., Estudo reológico de pastas de cimento contendo polímero superabsorvente e nano partículas de sílica, Dissertação de mestrado, PECC/UnB, Brasília, DF, Brasil, 2017.

[16] LYRA, J.S., Estudo da influência de policarboxilato comercial na hidratação, reologia e físico-química de superfície do cimento, Dissertação de mestrado, PMT/Poli/USP, São Paulo, SP, Brasil, 2010.

[17] ASSOCIAÇÃO BRASILEIRA DE NORMAS TÉCNICAS. NBR 16697: Cimento Portland - Requisitos. Rio de Janeiro, 2018.

[18] ASSOCIAÇÃO BRASILEIRA DE NORMAS TÉCNICAS. NBR 11579: Cimento Portland - Determinação da finura por meio da peneira $75 \mu \mathrm{m}$ (n 200). Rio de Janeiro, 2012.

[19] ASSOCIAÇÃO BRASILEIRA DE NORMAS TÉCNICAS. NBR 16372: Cimento Portland e outros materiais em pó - Determinação da finura pelo método de permeabilidade ao ar (método de Blaine). Rio de Janeiro, 2015.

[20] ASSOCIAÇÃO BRASILEIRA DE NORMAS TÉCNICAS. NBR 16607: Cimento Portland - Determinação dos tempos de pega. Rio de Janeiro, 2018.

[21] ASSOCIAÇÃO BRASILEIRA DE NORMAS TÉCNICAS. NBR 7218: Cimento Portland - Determinação da resistência à compressão de corpos de prova cilíndricos. Rio de Janeiro, 2019.

[22] ASSOCIAÇÃO BRASILEIRA DE NORMAS TÉCNICAS. NBR NM 18: Cimento Portland - Análise química - Determinação de perda ao fogo. Rio de Janeiro, 2012.

[23] ASSOCIAÇÃO BRASILEIRA DE NORMAS TÉCNICAS. NBR NM 15: Cimento Portland - Cimento Portland - Análise química - Determinação de resíduo insolúvel. Rio de Janeiro, 2012.

[24] ASSOCIAÇÃO BRASILEIRA DE NORMAS TÉCNICAS. NBR NM 16: Cimento Portland - Análise química - Determinação de anidrido sulfúrico. Rio de Janeiro, 2012.

[25] ASSOCIAÇÃO BRASILEIRA DE NORMAS TÉCNICAS. NBR NM 11-2: Cimento Portland - Análise química - Determinação de óxidos principais por complexometria. Parte 2: Método ABNT. Rio de Janeiro, 2012. 
[26] ASSOCIAÇÃO BRASILEIRA DE NORMAS TÉCNICAS. NBR NM 13: Cimento Portland - Análise química - Determinação de óxido de cálcio livre pelo etileno glicol. Rio de Janeiro, 2013.

[27] ASSOCIAÇÃO BRASILEIRA DE NORMAS TÉCNICAS. NBR NM 17: Cimento Portland - Análise química - Método de arbitragem para a determinação de óxido de sódio e óxido de potássio por fotometria de chama. Rio de Janeiro, 20132.

[28] JENSEN, O. M.; HANSEN, P. F. "Water-entrained cement-based materials I. Principles and theoretical background". Cement and Concrete Research, v. 31, n. 6, p. 647-654, 2001

[29] NETO, W. N. A., Caracterização de polímero superabsorvente para mitigação de retração autógena em microconcretos de alta resistência, Monografia de Projeto Final, ENC/UnB, Brasília, DF, Brasil, 2014.

[30] RILEM. Application of superabsorbent polymer (SAP) in concrete construction: state of the art report technical committee TC-225-SAP., London: Springer, p. 167, 2012.

[31] FRIEDRICH, S.V., Superabsorbent polymers (SAP), State of the art report of RILEM technical committee 225-SAP, RILEM, 2012.

[32] AMERICAN SOCIETY FOR TESTING AND MATERIALS. Standard Practice for Measuring Hydration Kinetics of Hydraulic Cementitious Mixtures Using Isothermal Calorimetry. ASTM C 1679 - 08, 2008. [33] BERRA, M., CARASSITI, F., MANGIALARDI T., PAOLINI, A.E., SEBASTIANI, M. "Effects of nanosílica addition on workability and compressive strength of Portland cement pastes", Construction and Building Materials, v. 35, p. 666-675, 2012.

[34] LOTHENBACH, B.; SCRIVENER, K.; HOOTON, R.D., "Supplementary Cementitious Materials", Cement and Concrete Research, v. 41, pp. 1244-1256, Dec. 2011.

[35] ZUNINO, F.; SCRIVENER, K. "The influence of the filler effect on the sulfate requirement of blended cements." Cement and Concrete Research, v. 126, n. August, p. 105918, 2019.

[36] ZUNINO, F.; SCRIVENER, K. "Factors influencing the sulfate balance in pure phase C3S/C3A systems." Cement and Concrete Research, v. 133, n. January, p. 106085, 2020.

[37] FRAGA, Y.S.B., Efeito da Ultrasonicação da Sílica Ativa e da Nanosílica na Microestrutura das Pastas Ternárias de Cimento, Dissertação de Mestrado, ENC/UnB, Brasília, DF, Brasil, 2019.

[38] KANG, S.; HONG, S.; MOON, J., "Importance of drying to control internal curing effects on field casting ultra-high performance concrete", Cement and Concrete Research, v. 108, pp. 20-30, Jun. 2018.

\section{ORCID}

Lívia Borba Agostinho

Juliana Gonçalves Borges

Eugênia Fonseca da Silva

Daiane Vitória Machado Ramos Cupertino https://orcid.org/0000-0002-7546-1499

https://orcid.org/0000-0001-9075-4591

https://orcid.org/0000-0001-8097-6107

https://orcid.org/0000-0003-1322-9472 